\title{
Research and Design of Precision Optical Interference Methane Concentration Detection System
}

\author{
Yanyu Zhang \\ Hunan Railway Professional Technology College, ZhuZhou Hunan 412001, China
}

\begin{abstract}
Currently, the stable optical interference type methane detector performance, long life, but due to manual reading, the low degree of automation, before the test, in order to prevent the emergence of large temperature changes in pressure calibration zero drift, scale values and must be Location temperature measured close to the inlet Lane, pinch release methane suction ball cleaning chamber, then zeroing. This device is not intuitive readings, human error is large enough to hold the data. Because of this, the use of image analysis and information processing, is proposed as a photoelectric conversion element using CMOS non-contact measurement, CMOS output signal after analog-digital conversion become high-speed digital image data to achieve CMOS digital image data by the DSP collection, storage, and image data for interference analysis algorithms, and ultimately to achieve high-precision measurement of methane concentration.
\end{abstract}

KEYWORD: Light interference methane detector; non-contact measurement; zero drift; digital signal processing

\section{INTRODUCTION}

Methane detection is mainly to detect the concentration of methane in air. Current application of portable methane detector is mainly divided into thermal conductivity methane detector (Ye Weilin et al, 2012), infrared methane detector, thermal catalytic methane detection alarm, optical methane detector, and so on (Qiao Xueguang et al, 2009). At present, optical interference type methane detector has stable performance, long service life, but due to the use of artificial reading, low degree of automation (Huang Youwei et al, 2006), before the test, scale value calibration must be in to be measured and the location is close to the temperature of the into the airway, pinch the suction chamber cleaning methane balloons, and zero calibration (Zheng Chuantao et al, 2014) (Feng Hongyan et al, 2010), anti stop due to temperature changes in air pressure larger and zero drift phenomenon, reading is not intuitive, human error is larger, can store data (Yan Yi, 2013). Based on this. In this paper, the image analysis and information processing, the CMOS as a photoelectric conversion element of non-contact measurement, CMOS output signal after a / D conversion become high-speed digital image data, through the DSP Implementation of CMOS digital image data acquisition, storage, and image data of the algorithm for interference analysis, the ultimate realization of high precise measurement of methane concentration.

\section{OPTICAL INTERFERENCE DETECTION MECHANISM}

The refractive index of different gas is different from the principle of the optical methane detector. Fringes generated in the air and methane room at the same time, under the same conditions, filled with the same density of fresh air through the instrument enters the calibration, when in the sample gas chamber filled with methane, due to the refractive index of different, cause the changes of optical path, interference fringes will produce and filled with methane concentration on the corresponding offset, this offset can be used to indicates that methane concentration. The higher the concentration, the greater the shift. Through test eyepiece interference fringe movement distance, according to the moving interference fringes displacement after the methane concentration scale value, measured methane concentration.

The refractive index of pure air gas is $n_{0}$, and the refractive index of pure methane gas is $n_{1}$. If the sample gas chamber is filled with methane gas with the concentration of $\mathrm{X}$, the refractive index: 
$n_{2}=n_{1} \times \frac{x}{100}+n_{0} \times \frac{100-x}{100}$

While the standard chamber for the pure air, so $\delta=2\left(n_{2}-n_{0}\right) l$ is the optical path of two optical path difference, where $l$ is the length of gas chamber (Fig. 1).

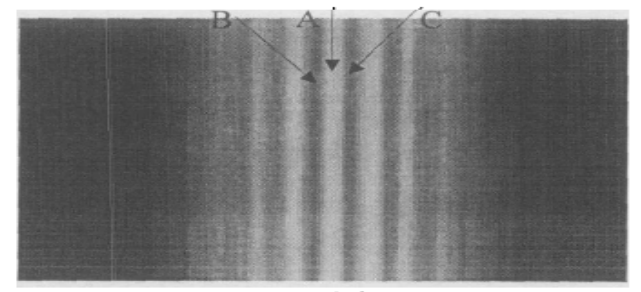

Fig. 1 Interference fringe pattern

The interference fringe pictured methane detector shows, it is produced by white light, white light fringe center is zero, is on both sides of several color stripes and two zero black dark stripes. We believe that the zero order white lines for white baseline, assuming sample gas chamber without charge in methane, white pixels corresponding to the A baseline. When the sample gas chamber containing methane, interference fringe shift, two zero order black dark lines correspond to the pixels were $\mathrm{B}$ and $\mathrm{C}$, then is a fringe spacing, we only need to identify baseline offset corresponds to the pixel location, remember to $\mathrm{D}$, you can draw stem involving moving striation spacing number

$N=\frac{D-A}{C-B}$

According to the principle of interference fringes, moving a fringe spacing corresponding to the optical path difference variation is $\lambda$, i.e.

$$
N \lambda=2\left(n_{1} \times \frac{x}{100}+n_{0} \times \frac{100-x}{100}-n_{0}\right) l
$$

The equation of methane concentration is obtained:

$$
x=100 \times \frac{\lambda}{2 l\left(n_{1}-n_{0}\right)} \frac{D-A}{C-B}
$$

Where $\lambda$ is the wavelength of the light source.

The refractive index is related to temperature and pressure, so the change of ambient temperature and pressure will affect the measurement results. As long as into methane gas composition and the air chamber of the gas composition is not the same, interference fringe will move, interference fringe movement and gas composition of a relationship. Therefore, optical interference methane detector was affected by the outside gas. In order to ensure the accuracy of measurement of optical interference methane detection instrument, are equipped with external absorption of carbon dioxide and water vapor absorbent. In addition, if the difference in temperature test place light interference methane detector with the use of a site, or the measured gas oxygen, the measurement results will be affected.

\section{CMOS IMAGE ACQUISITION SYSTEM}

Using this module the core component of the CMOS camera is ov OV6620 camera; pre camera output image data stored by IDT Corporation FIFO memory, the working voltage of the two are $5 \mathrm{~V}$, data input and IDT7205 OV6620 image data can be of direct connection, but processor IO voltage $3.3 \mathrm{~V}$, in the design should note data can not be directly connected.

OV6620 camera output timing is shown in Fig. 2, VSYN represents the beginning of a frame image, HREF signal is high at this time the image data is valid, while the PCLK signal for pixel signal, rising edge of PCLK.

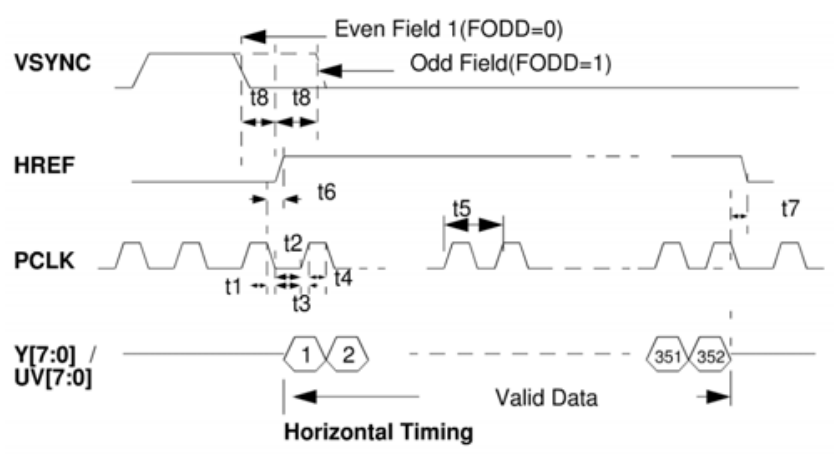

Fig. 2 OV6620 camera output timing

Image data is valid. This can be analyzed, only between two VSYN, and the HREF is high, the output of the corresponding PCLK is effective image data. Therefore, the image acquisition module using a three input and gate to the href and PCLK and processor control and operation, get the right to write timing ENW.

The camera on the electricity, they continue to have an interrupt signal and image signal output. Among them, before the arrival of each frame image, VSYN will have a pulse signal. From Fig. 3, you can determine the arrival of a frame image by capturing the rising edge of VSYN.

VSYN

Fig. 3 pulse signal

By default, the camera capture image resolution is 356*292. In the CMOS image acquisition system, 
when the ENW control signal is set, the system will automatically begin to read the FIFO camera output data. In this way, the image information will be in the FIFO cache area in order to store data (Fig. 4).

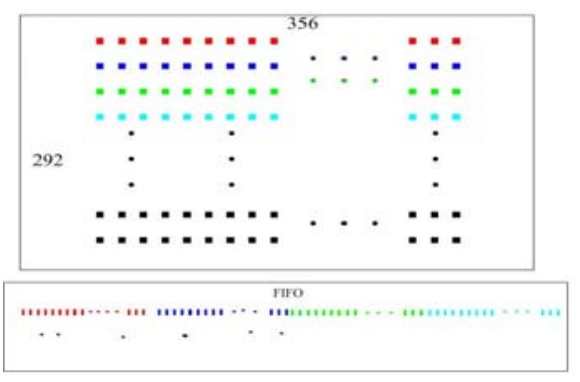

Fig. 4 FIFO cache area

Because the storage space of the buffer is limited, each piece of $8192 * 8 \mathrm{bit}$, a total of two blocks can be stored in $16384 * 8$ bit. So, $16384 / 356$ can store about 46 rows of data by default in FIFO. On the other hand, taking into account the first in the first out of the FIFO features, so as to avoid redundant data into the FIFO buffer, the need for the arrival of the second VSYN signal when the ENW signal is low, shielding the image information to the buffer to write. When the CMOS data collected by the data written in FIFO, the core development board can simply by controlling the RD signal to the gray level signal. Timing is shown in Fig. 5.

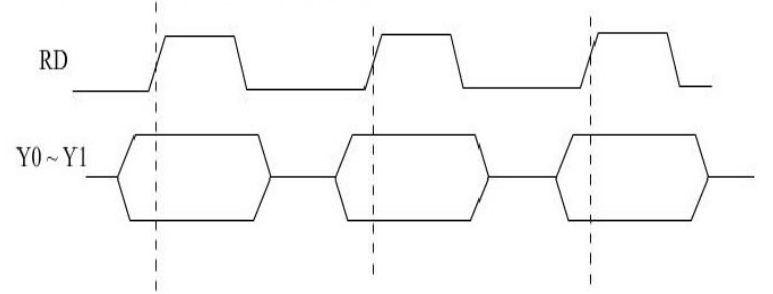

Fig. 5 Timing

DSP core development board software code is mainly composed of peripheral resource initialization module, core processing module, interrupt response module, image scan analysis module, serial communication and alarm control module. In order to provide the service for each module of the main program, the initialization of the peripheral resources is carried out by using the pin resources in the development board. Core processing module is the core of the program operation, the difference between the system standby and processing work to make different state, to ensure the normal operation of the system and the low power consumption demand. Interrupt response module is the key of the core development board and COMS image acquisition system communication, including VSYN and HREF two interrupt source. Accurately for each image arrival and a picture of the arrival of each line of information to identify, help the main program for image analysis and processing, on the other hand, the system can also reduce the need for storage space. Image scanning and analysis module is the core of the whole application, the recognition and calculation of the fringe information is completed. Serial communication and alarm control module is the methane detector system is more perfect, so as to achieve the purpose of precision control. Serial communication for the DSP core board and PC communications to provide the possibility, so that the core development board to collect the methane concentration of information real-time transmission to the PC side, and the way to draw a curve graph. The alarm module mainly uses TMS320F28027 to provide efficient ePWN wave to control the buzzer alarm.

\section{SOFTWARE SYSTEM DESIGN}

In the processing of each line, the zonal distribution of the interference fringes is the key to correctly judge the white base and the zero order. In the interference image, the gray value of the same fringe is very close, and the gray value between the adjacent stripes is very different. So it can be judged that the difference between the adjacent pixels can be distinguished by the difference between the adjacent pixels. In the actual sampling CMOS, there will be some random phenomena, such as the dark lines in a white pixel. Because it is needed to compare the number of pixels in the edge of the fringe, the effect of this random pixel is excluded. The process flow chart of fringe differentiation process is shown in Fig. 6.

\section{TEST AND CONCLUSION}

The current system basic achieve the expected goal: real-time acquisition of methane interference fringe information, analysis calculation of methane concentration, control and alarm, and real-time information of methane concentration in the curve mode display in the Borland $\mathrm{C}++6.0$ interface. In order to facilitate the analysis and processing, this paper collected the image of the camera through the serial port to PC, in order to store the .Bmp format. Fig. 7 gives a picture of the fringe information collected by the camera.

In this paper, the collected fringe information is analyzed and processed on Matlab. The results show that the width of the stripe is about 80 pixels, and the height is 12 pixels. At the same time we will have a horizontal stripes in the plot way to draw out as shown in Fig. 8. Where there is a wide range of areas where the information is located in the fringe pattern. 


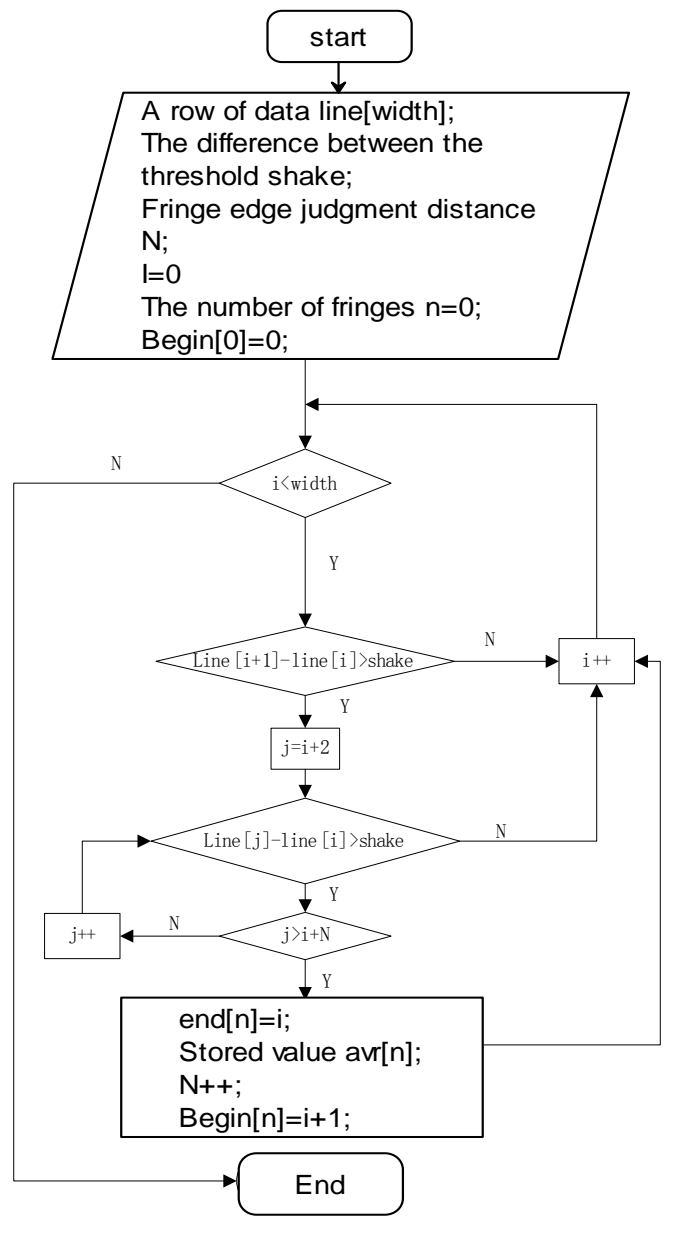

Fig. 6 Flow chart of fringe differentiation process

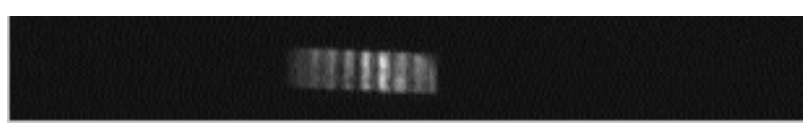

Fig. 7 fringe information

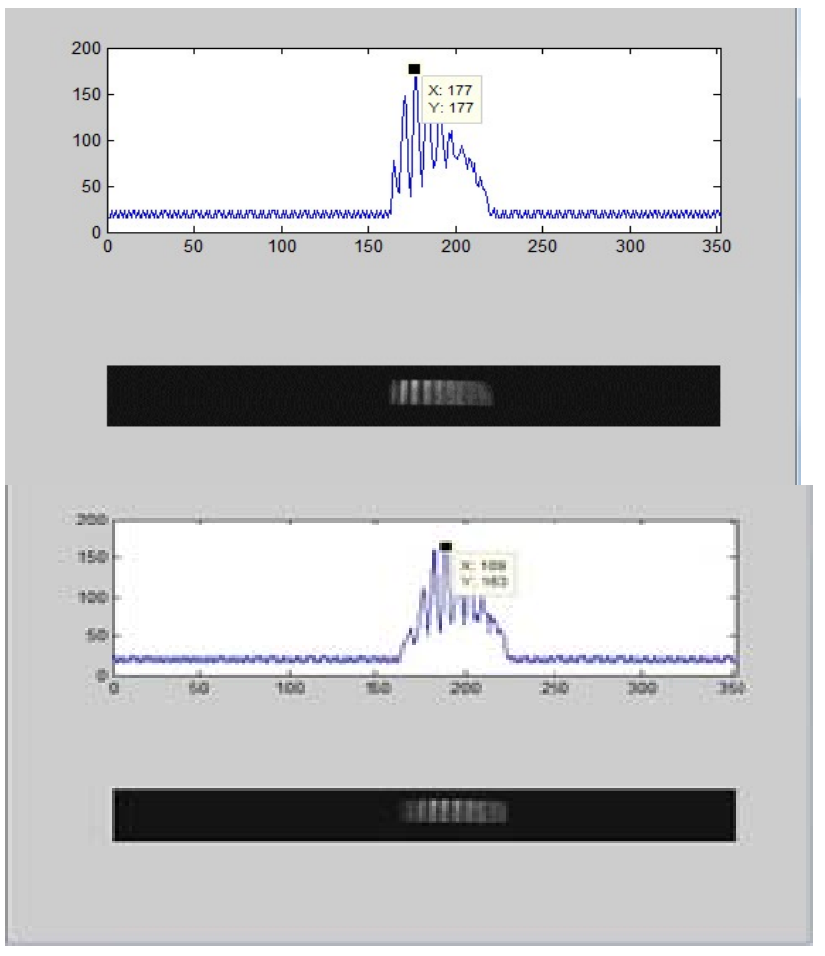

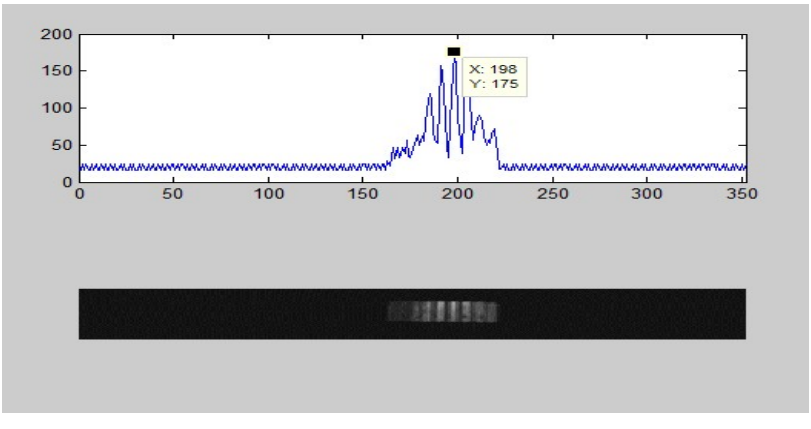

Fig. 8 horizontal stripes

Through the test, it is found that there are two distinct peaks in each line, and the distance is relatively constant. So when the algorithm is realized, the two peak position is used to determine the central bright pattern, the biggest advantage of this method is that it can avoid the error to determine the location of the white base line due to the high gray level of the pixels of the accident. The following picture gives a series of fringe moving photos, and corresponding in MATLAB show the gray information curve, also in the curve marked the location of the peak.

\section{REFERENCES}

Feng Hongyan, Wang Renbao. Laser spectroscopy methane monitoring system based on digital signal processor [J]. Optoelectronic lasers, 2010, 21(01): 38-41.

Huang Youwei, Tong Minming, Ren Zihui. Study on a new method of gas concentration detection using thermal conductivity sensor [J]. Journal of sensing technology. 2006.19(04).973-975.

Qiao Xueguang, Wang Jia, Jia Zhenan, et al. Experimental study on optical fiber $\mathrm{CH} 4$ gas sensor [J]. Optoelectronic lasers, 2009, 20(07): 851-854.

Yan Yi. Research on optical fiber methane monitoring technology based on Ant Algorithm [D] Master Thesis of Anhui University of Science And Technology.2013.

Ye Weilin, Lv Ruihong, Song Fang, et al. Two quantization suppression of voltage noise in methane detection and its experimental study [J]. Optoelectronic lasers, 2012, 23(06): 1140-1145.

Zheng Chuantao. Wang Yiding Ye Weilin. Stability test and temperature compensation of medium infrared methane detector [J]. Journal of Optics. 2014 34(02).1-5. 